\section{Isolated Gastric Tuberculosis: A Case Report}

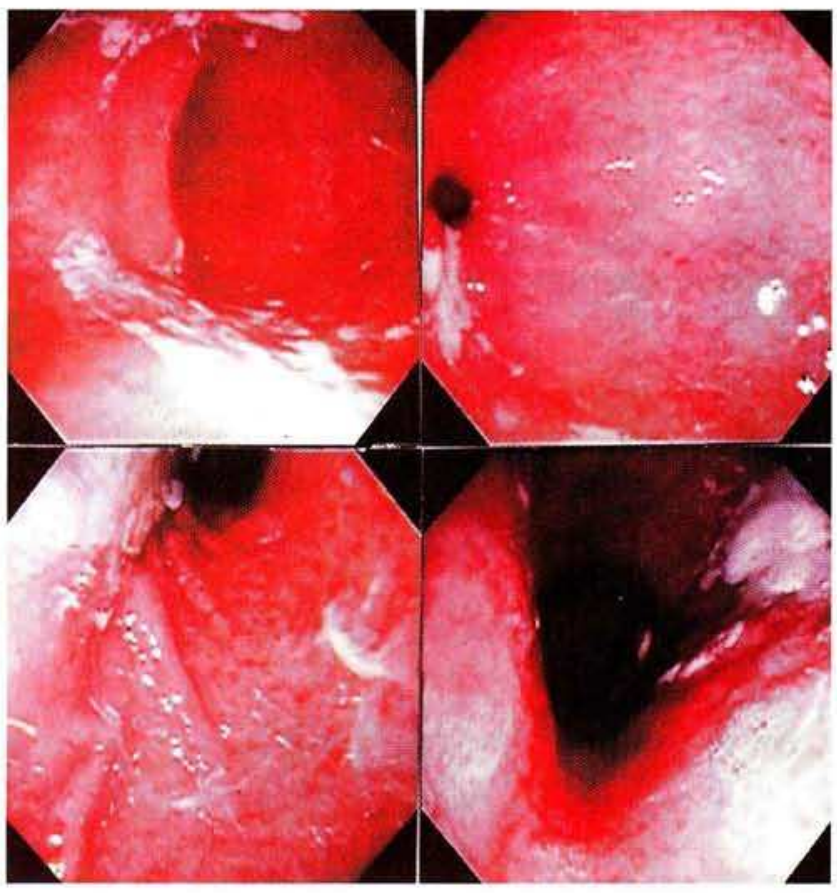

Figure 1: Endoscopic view of the stomach with atrophic mucosa and signs of diffuse chronic inflammatory activity. (Videoendoscope Olympus CV-1)

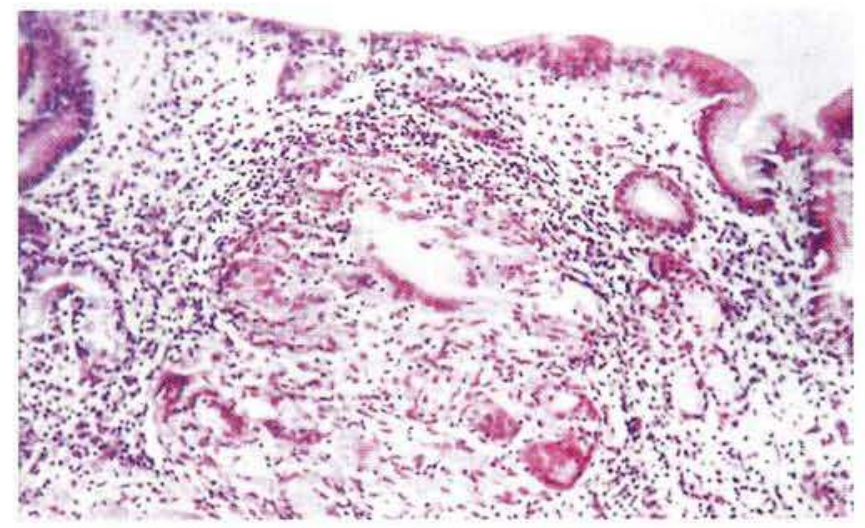

Figure 2: Light photomicrograph of the gastric mucosa showing a non-caseating granuloma with giant cells, surrounded by a lymphocytic rim. The superficial epithelium is preserved. (Hematoxilin and Eosin, $\times 100$ )

Gastric involvement secondary to pulmonary tuberculosis (TB) is rare $(1-3)$. Even more extraordinary is the finding of isolated TB, without symptoms of specific disease in other organs or systems $(2,4)$.
We present a case of gastric tuberculosis in a 52-year-old woman who was submitted to an upper GI endoscopy for dyspeptic symptoms, anorexia and weight loss. On endoscopy a diffusely atrophic gastric mucosa, a generalized oedema, foci of acute inflammation with petechial lesions and erosions, and abundant whitish, thick and viscous mucous adhering to the walls were found (Figure 1). Esophagus, duodenal bulb and duodenum were normal. Histologically, (Figure 2) an epitheliod cell granuloma with Langhans cells and few acid-fast bacilli was found on a biopsy sample. The diagnosis of gastric tuberculosis was confirmed by juice aspiration and cultures in Lowenstein specific medium. Studies carried out in order to identify active tuberculous disease in any other location were negative. Blood tests for AIDS, and Helicobacter pylori on the gastric mucosa, were negative. The patient was treated with antituberculous therapy, with a good clinical evolution. Past medical history was significant for pulmonary tuberculosis 30 years ago with negative periodical controls thereafter.

Therefore this is one of the rare cases of isolated gastric tuberculosis with no evidence of active tuberculosis in any other organs. The majority of the gastric tuberculous cases are secondary to other tuberculous lesions (2) and in the literature only three cases reported $(2,4,5)$ isolated tuberculous lesions. In conclusion we believe that in patients with endoscopic evidence of diffuse chronic inflamatory activity and history of previous TB disease, gastric tuberculosis should always be kept in mind in the differential diagnoses. In view of these findings we consider it necessary to perform multiple endoscopic biopsies in different areas of the gastric mucosa, since the granulomatous lesions are usually scattered with areas of chronic unspecific inflammation in between.

\section{J. Perez-Piqueras*, S. Coca, C. Silva, D. Martinez,}

J. Peralba, M. Moreno

Hospital Universitario del Aire

(Servicio de Aparato Digestivo)

Arturo Soria 82

28027 Madrid, Spain

\section{References}

1. Klimach $O E$, Ormerod $L P$; Gastrointestinal tuberculosis: A retrospective review of 109 cases in a District General Hospital. Quarterly J. Med. 1985; 221: 569-578.

2. Subei I, Attar B, Schmitt $G$ et al.: Primary Gastric Tuberculosis: A case report and literature review. Am. J. Gastroenterol. 1987: 82: 769 -772 .

3. Palmer ED: Tuberculosis of the stomach and the stomach in tuberculosis. A review with particular reference to gross pathology and gastroscopic diagnosis. Am. Rev. Tuberc. 1950; 61: 116-133.

4. Misra RC, Agarwall SK, Prakash P et al.: Gastric tuberculosis. Endoscopy 1982; 14: 235-237.

5. Tromba $J L$, Inglese $R$, Rieders $B$ et al.: Primary gastric tuberculosis presenting as pyloric outlet obstruction. Am. J. Gastroenterol. 1991 12: $1820-1822$ 